\title{
Anabases
}

ANABASES Traditions et réceptions de l'Antiquité

6 | 2007

Varia

\section{Villoison et la redécouverte du dialecte tsakonien}

\section{Étienne Famerie}

\section{(2) OpenEdition}

\section{Journals}

Édition électronique

URL : http://journals.openedition.org/anabases/3475

DOI : 10.4000/anabases.3475

ISSN : 2256-9421

\section{Éditeur}

E.R.A.S.M.E.

Édition imprimée

Date de publication : 1 octobre 2007

Pagination : 235-248

ISSN : 1774-4296

\section{Référence électronique}

Étienne Famerie, «Villoison et la redécouverte du dialecte tsakonien », Anabases [En ligne], 6 | 2007, mis en ligne le 01 janvier 2012, consulté le 20 octobre 2019. URL : http://journals.openedition.org/ anabases/3475; DOI : 10.4000/anabases.3475

Ce document a été généré automatiquement le 20 octobre 2019

(c) Anabases 


\title{
Villoison et la redécouverte du dialecte tsakonien
}

\author{
Étienne Famerie
}

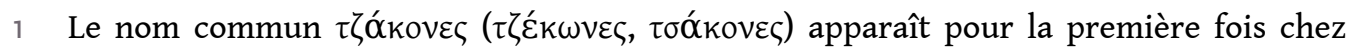
Constantin Porphyrogénète (Cérém., I, p. 695 Bonn), pour désigner une catégorie de soldats " appauvris", incapables d'assurer les frais inhérents à leur service. Le terme n'a alors aucun rapport ni avec une région déterminée de l'empire byzantin, ni avec le dialecte «tsakonien». Les "tsakones " sont une catégorie parmi d'autres de soldats auxiliaires affectés à la garde des forteresses situées aux endroits stratégiques du territoire (frontières, côtes, défilés, etc. ${ }^{1}$ ). Même s'ils apparaissent d'abord comme des militaires déclassés, ils constituent une pièce importante dans la surveillance des provinces; complémentaires de la cavalerie et de l'infanterie régulière, ces fantassins légers sont les mieux placés pour les missions de police locale, d'observation, voire d'espionnage. L'institution a aussi évolué au fil du temps : au xiII 'e siècle, par exemple, le terme recouvre une réalité plus large et désigne des soldats auxiliaires recrutés pour compléter le système de défense, y compris dans la marine et dans la garde impériale ${ }^{2}$.

2 À la fin $\mathrm{du} \mathrm{XvI}^{\mathrm{e}}$ siècle, on a redécouvert en Occident l'existence d'un dialecte pratiqué par des populations installées dans l'est du Péloponnèse ${ }^{3}$. Les sources de l'époque font

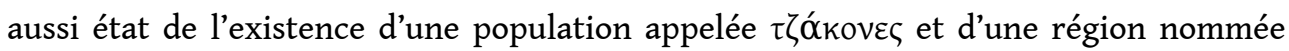

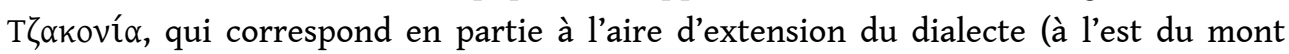
Parnon, d'Argos au cap Malée, mais aussi en Laconie, à Sparte et Mistra). Entre le XVII et le $\mathrm{xx}^{\mathrm{e}}$ siècle, les recherches se sont multipliées, sans guère se soucier de rassembler tous les témoignages et sans prendre en compte la chronologie relative des sources; de plus, ces recherches ont longtemps reposé sur une erreur de méthode. Faute d'avoir

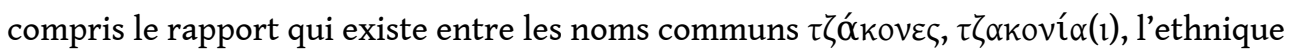

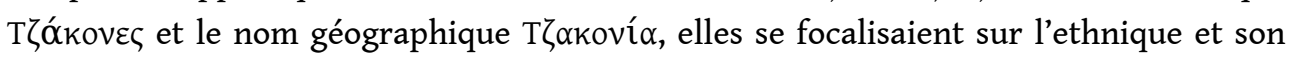
étymologie. L'histoire de ces recherches est intéressante en soi, car elle reflète les tensions nationalistes qui se sont exprimées dans les Balkans jusqu'au $\mathrm{xx}^{e}$ siècle $^{4}$. Je me bornerai ici à en donner une illustration. 
Des érudits slaves, se fondant sur l'assimilation Tzaconia et Sclavonia, qu'on trouve dans des sources vénitiennes, ont tenté de démontrer que le tsakonien était un dialecte slave. Mais les progrès des études de phonétique historique du grec et de ses dialectes ont vite montré qu'il s'agissait à coup sûr d'un parler grec aux traits archaïques, proche du dorien. Dès lors, il était tentant, pour les Grecs et les philhellènes, d'assimiler les

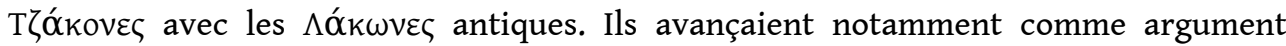
l'origine du dialecte et son aire d'extension, la Tsakonie, que les sources les plus tardives limitent à l'ancienne Cynourie. Cette assimilation se heurtait cependant à de sérieuses objections. D'une part, le problème était mal posé : historiquement, les $\tau \zeta$ ókoveৎ n'avaient pas de lien exclusif avec l'est du Péloponnèse ; autrement dit, les tsakones du $\mathrm{x}^{\mathrm{e}}$ siècle n'étaient pas les habitants de la Tsakonie, une région dont le nom

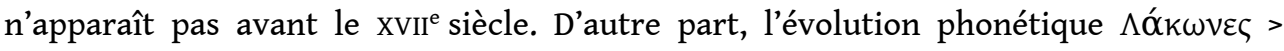

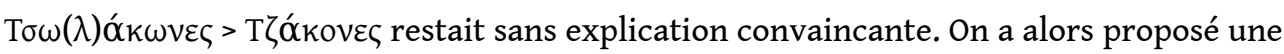

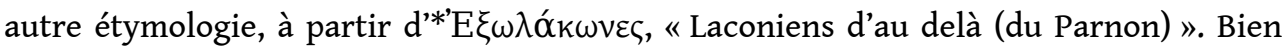

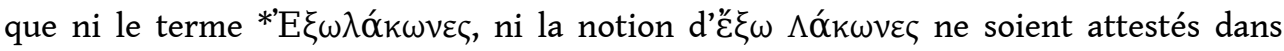
aucune source, l'hypothèse a eu la vie longue, agrémentée d'interprétations diverses de l'élément $\varepsilon \xi \omega$ (« Laconiens de l'extérieur, de la campagne », « Laconiens étrangers à la communauté, païens ", etc.).

4 S.C. Caratzas a, le premier, abordé la question de l'étymologie sur des bases plus solides. Conscient que l'explication géographique («laconienne ») aboutissait à une impasse, il a recueilli l'ensemble des testimonia et est parti de la forme la plus ancienne (le nom

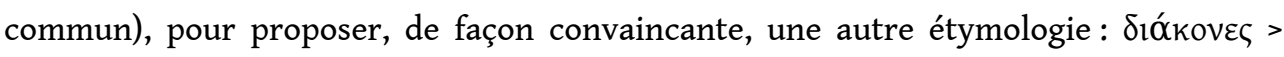

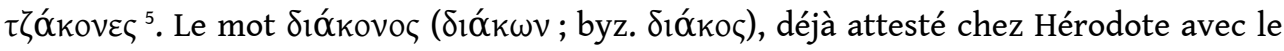
sens de "serviteur", s'est maintenu en grec byzantin, dans un contexte profane (" auxiliaire ») et religieux (" diacre »). Le témoignage de Constantin Porphyrogénète

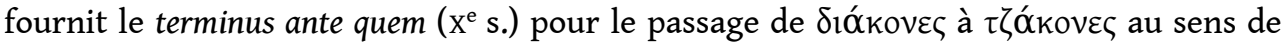
« soldats auxiliaires".

5 Le terme $\tau \zeta \alpha$ kovía désigne un "groupe de soldats auxiliaires", puis leur lieu d'installation. L'empire byzantin comptait de nombreuses $\tau \zeta \alpha$ koví $\alpha$ l, qui ont donné lieu à un grand nombre de toponymes ${ }^{6}$, ce qui explique en partie l'apparente confusion dans les sources les plus anciennes. Le passage de $\tau \zeta \alpha$ koví $\alpha$ à $T \zeta \alpha$ koví $\alpha$ pour désigner une région particulière s'explique, selon Caratzas, par la résistance acharnée qu'ont opposée les Slaves du centre et de l'est du Péloponnèse par rapport à ceux du nord, dont l'assimilation a été plus rapide. En d'autres termes, la Tzaconie tirerait son nom de la permanence remarquable et de l'importance des $\tau \zeta \alpha$ kovíal présentes dans cette région; ainsi s'explique que des sources tardives ont pu assimiler la Tzaconia avec la Sclavonia. En particulier, sous la domination vénitienne (1685-1715), la Tsakonie devint une des quatre entités administratives de la Morée. Elle correspondait alors au territoire situé à l'est de l'Eurotas. À la suite du dépeuplement de la péninsule du cap Malée, la Tsakonie s'est réduite, depuis la fin du XVIII ${ }^{\mathrm{e}}$ siècle au moins, à une région de l'éparchie de Cynourie (entre Agios Andreas et Leonidio). Elle compte aujourd'hui une dizaine de villages ${ }^{7}$.

Jean-Baptiste-Gaspard d'Ansse de Villoison (1750-1805) est le premier à avoir porté un intérêt philologique au dialecte tsakonien. L'éditeur du célèbre Venetus A de l'Iliade 
avait été chargé de parcourir les bibliothèques de la Grèce et de l'Archipel en vue d'acquérir de nouveaux manuscrits d'auteurs anciens ${ }^{8}$. Lors de son passage au monastère de Vatopedi (Athos), il reçoit l'information suivante :

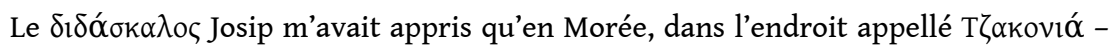

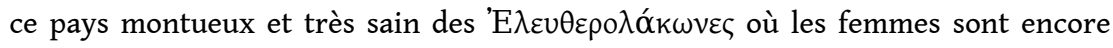

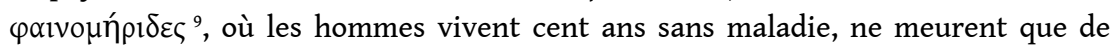

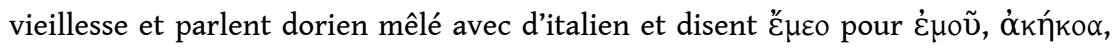

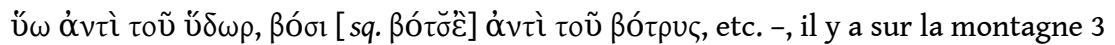

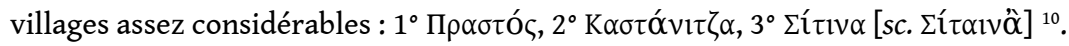

Constatant que sa quête de manuscrits est un échec, Villoison renonce à sa mission première et entreprend un voyage " archéologique " qui le conduit en Grèce centrale, dans le Péloponnèse, en Attique, dans les îles de l'Égée, à Smyrne et à Éphèse (1785-1786). Philologue de formation, helléniste curieux, il visite plusieurs sites, y copie des inscriptions (notamment dialectales), mais s'intéresse aussi à la Grèce de son temps.

8 Au terme de son voyage, Villoison avait l'ambition de rédiger une étude comparée de la Grèce ancienne et moderne, un projet encyclopédique qui est resté à l'état d'ébauche, de fiches pourrait-on dire, à la suite de son décès inopiné en $1805^{11}$ :

La relation de mon voyage, que je publierai un jour en plusieurs volumes, est trop étendue pour que j'en puisse renfermer l'abrégé dans un seul mémoire: elle me fournira une suite nombreuse de dissertations. Je ne parlerai, dans celle-ci, que des inscriptions que j'ai eu le bonheur de découvrir; les autres rouleront sur le mont Athos, sur le singulier genre de vie de ses habitans, sur les monastères grecs, sur leurs bibliothèques, sur les différens monumens que j'ai vus dans le Levant, sur la langue, les mœurs, les usages, les cérémonies religieuses, les habillemens, l'agriculture, le commerce, la marine, les maladies des Grecs modernes comparés avec les anciens, sur les Tzaconiens, chez lesquels j'ai retrouvé en partie la langue des anciens Doriens, le dialecte de Pindare et de Théocrite ${ }^{12}$.

9 Le témoignage de Villoison sur le dialecte tsakonien est conservé dans son journal de voyage (environ 160 mots ${ }^{13}$ ), qu'il a utilisé dans une longue note figurant dans les Prolegomena à son édition de l'Iliade ${ }^{14}$ :

Naupliam inter \& Epidaurum, Limeram, sive Monembasiam, vicatim in praeruptis montibus habitant Tzacones, ab antiquis Laconibus oriundi. De his vide Cangium, in

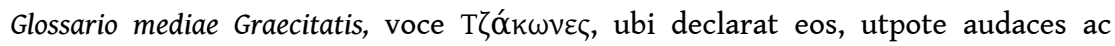
fortes, a Michaele Palaeologo Imperatore, non in militiam modo, sed \& in custodiam

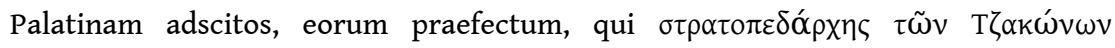
dicebatur, in officialium Palatinorum numerum fuisse relatum. Minime confundendi sunt cum Mainotis, a Maina, prius dicta Messa, vocatis \& inter

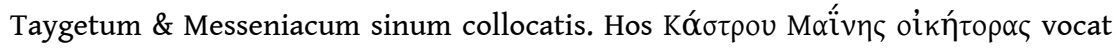
Constantinus Porphyrogenitus, De administrando imperio, c. 50, p. 180 ed. Meursii, ubi

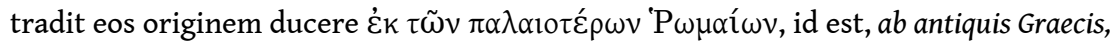

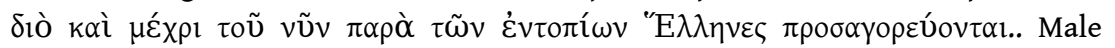
ibidem Meursius vertit, ab antiquis Romanis, 'P $\omega \mu \alpha i ́ \omega v$; nam post Romani imperii sedem translatam Constantinopolim, Graeci semper, \& etiam nunc hodie, vocati sunt ' $\mathrm{P} \omega \mu \alpha \tilde{i} о$, \& eorum lingua $\mathrm{P} \omega \mu \alpha \ddot{k} \kappa \eta$, ut distinguatur ab antiqua, quam vocant

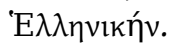

Tzaconum, qui multo puriorem quam ipsi Mainotae servaverunt linguam, tria sunt hodie parva oppida, nostris geographis \& itinerariorum scriptoribus ignota, Prasto (forte antiqua urbs Prasiae), Kastanitza \& Sitina; quorum duobus primis quidem 400 circiter domus, tertio vero, centum comprehenduntur. His adde quosdam uiculos, qui dicuntur Platanos, Karakovouni, Hagia Anna, seu, ut efferunt, Haj' Anna (Sancta Anna), Hagio-Petro, Castri, \&c. Hi veluti Graeciae Helvetii habendi sunt, utpote boni, ingenui, candidi, laboris patientes, veritatis \& hospitum amicissimi, 
robusti, animosi, \& usque ad centum annos vitam perducunt sine morbis \& medicis ; quorum tamen unus nuper irrepsit in Prasto, quod est Tzaconiae caput, \& in quo reperiuntur monachi Graeci, quidam $\delta 1 \delta \alpha ́ \sigma \kappa \alpha \lambda o \varsigma$, id est, litterarum Graecarum, \& primorum antiquae linguae grammaticae elementorum magister, \& nonnulli mercatores, qui interdum Constantinopolim negotiaturi petunt. Tzaconum mulieres procerae, formosae, valentes \& sanae, solae fere in Graecia \& in toto Oriente interioribus feminalibus, sive subligaculis, non utuntur.

Cum plurimis Tzaconibus in Peloponneso, \& maxime Spartae \& Naupliae, collocutus sum ; \& Athenis sex hebdomadum spatio, quemdam ex iis conduxi, quo suggerente, Tzaconicae linguae, sive recentis Laconicae, grammaticam \& vocabularium a me edenda scripsi. In suis asperrimis \& fere inaccessis montibus multas antiquas voces \& formas Doricas caeteris Graecis recentioribus novas \& inauditas retinuerunt;

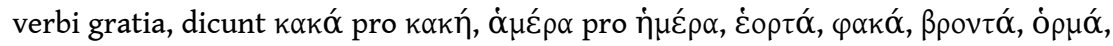

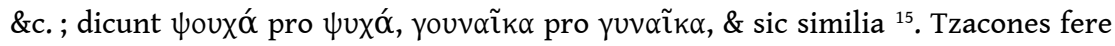

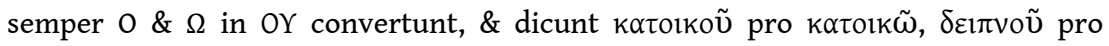

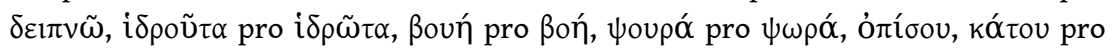

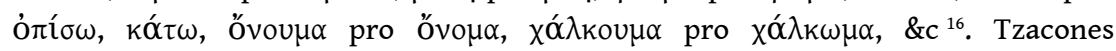

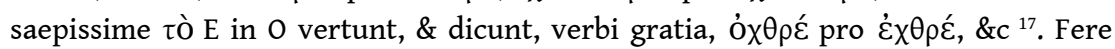

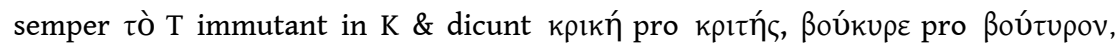

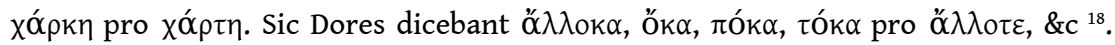

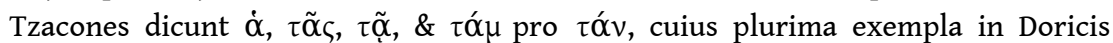
inscriptionibus, $\dot{\omega} \rho \alpha_{k \alpha} \alpha \varepsilon v, \& c ., \& c$. Sed maxime notandum est eos semper \& constantissime nominativum, ut vocativum inflectere, \& dicere ö́ $\xi \varepsilon, ~ o ̈ \lambda \lambda \varepsilon, \pi ı \rho \rho ́$,

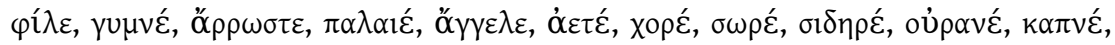

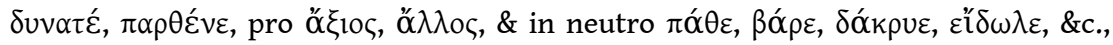

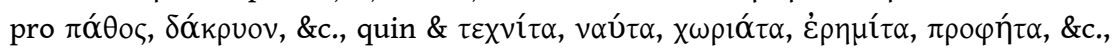
\&c., pro $\tau \varepsilon \chi v i ́ \tau \eta \varsigma, ~ v \alpha u ́ \tau \eta \varsigma, ~ \& c ., ~ \& c^{19}$. Multa veteris linguae vocabula in sermone vulgari usurpant, quorum sensum alii Graeci assequi non possunt. Sic dicunt $\pi$ opeí $\alpha$

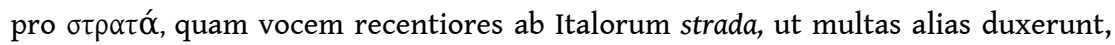

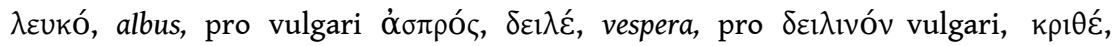

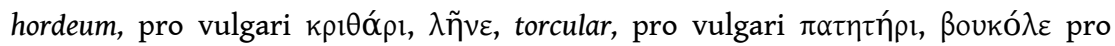

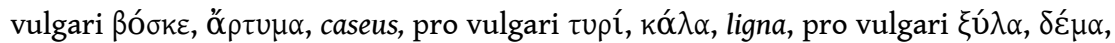

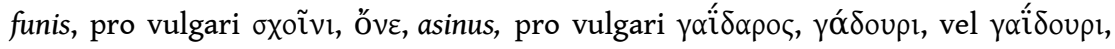

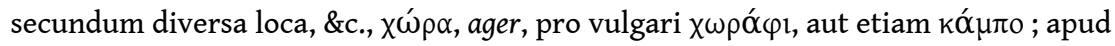

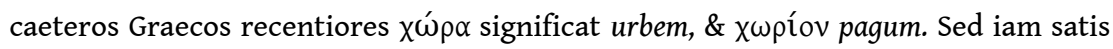
est exemplorum. Decimo sexto saeculo Symeon Cabasilas ex civitate Graeciae Acharnania, ut ipse dicit, Martino Crusio scribebat: Adhuc ex nostris illitteratis, alios Dorice, alios Attice, quosdam Aeolice, nonnullos Ionice, communi denique lingua alios

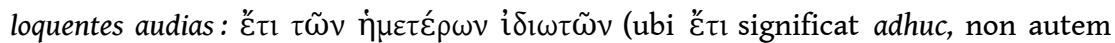

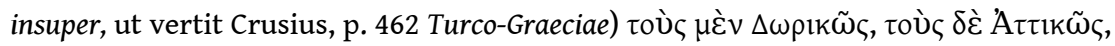

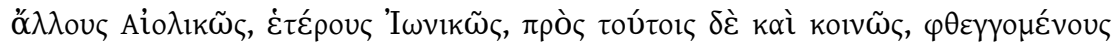

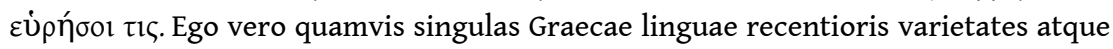
immutationes sedulo notaverim in omnibus Graeciae regionibus \& insulis, quas lustravi, nullum huc usque superesse deprehendi antiquarum dialectorum vestigium nisi apud Tzaconas, de quibus haec Martino Crusio, p. 489 Turco-Graeciae scribebat Gerlach: Omnes Graeci, quocumque locorum, se mutuo intelligunt, exceptis Ionibus (sic) qui in Peloponneso inter Naupliam et Monembasiam 14 pagos inhabitantes, antiqua lingua, sed multifariam in grammaticam peccante, utuntur; qui grammatice loquentem intelligunt, vulgarem vero linguam minime ${ }^{20}$.

10 Malgré les nombreuses recherches dont le tsakonien a fait l'objet depuis le XIX ${ }^{\mathrm{e}}$ siècle ${ }^{21}$, le témoignage de Villoison est intéressant à plusieurs égards.

11 Sûr de son érudition (il maîtrise la bibliographie sur le sujet, dirait-on aujourd'hui), il opère de savants rapprochements avec des termes dialectaux antiques et n'hésite pas à 
critiquer le jugement de ses prédécesseurs sur le caractère «barbare» du tsakonien. Pour l'helléniste, la découverte tenait du miracle : le tsakonien lui paraissait sortir du fond des âges. Se fondant sur Pausanias (III, 21-26), qui lui sert de guide de voyage, Villoison ébauche déjà une explication ethno-géographique du phénomène tsakonien ; il identifie le pays des Tsakones avec celui des anciens 'E $\lambda \varepsilon v \theta \varepsilon \rho \circ \lambda \alpha ́$ ó $\omega v \varepsilon \varsigma$ et tient ses habitants pour les héritiers de la vieille civilisation, eux qui parlaient toujours le dialecte de ses deux poètes favoris, Pindare et Théocrite. Il est le premier à donner un nom au dialecte (Tzaconica lingua, sive recens Laconica) et annonce rien moins que l'édition d'une grammaire et d'un vocabulaire du tsakonien ${ }^{22}$.

Il est surtout le premier à diffuser dans le monde savant un échantillon significatif et commenté de mots tsakoniens recueillis lors de conversations avec des locuteurs, ce qui lui cause d'ailleurs un certain embarras pour noter ce qu'il entend ${ }^{23}$. Le choix des mots retenus dans les Prolegomena (environ un tiers par rapport au journal) visant à mettre en relief la remarquable permanence d'un dialecte antique à travers le tsakonien, Villoison évite de mentionner des termes empruntés aux langues modernes ${ }^{24}$; il

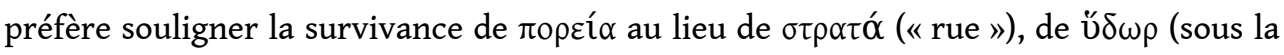
forme v́o) au lieu de vepó (" eau»), etc. Les contraintes typographiques ont pu aussi influencer le choix opéré ${ }^{25}$.

Le rôle pionnier de Villoison dans les études tsakoniennes a été souligné à maintes reprises, mais n'a guère reçu d'explication ${ }^{26}$. Il était peut-être, à son époque, l'un des seuls savants à pouvoir traiter du dialecte. D'une part, ses travaux sur la langue d'Homère (édition du lexique d'Apollonios Dyscole, du Venetus A et de ses scholies), sa familiarité avec des auteurs comme Hésychios et Eustathe, sa prédilection pour Pindare et Théocrite avaient éveillé son intérêt de philologue pour les dialectes grecs. Lors de son voyage en Grèce, la découverte d'inscriptions dialectales dans le Péloponnèse (en particulier à Épidaure) lui avait montré que le dorien n'avait pas été qu'un dialecte littéraire. D'autre part, ses recherches sur le grec appelé alors «vulgaire» et sa maîtrise de la langue (il voyage d'ailleurs sans interprète) lui avaient valu d'être le premier titulaire de la chaire de grec moderne à l'École des langues orientales vivantes ${ }^{27}$. Villoison était donc particulièrement bien armé pour mener la première étude sur le tsakonien. En cela, il était peut-être bien, selon l'expression de J.E. Sandys, « le dernier savant de la vieille école ${ }^{28} \%$.

14 À l'été 2005, j'ai visité avec une profonde émotion les trois villages mentionnés par Villoison, Prastos, Kastanitsa et Sitena, tenant en main l'édition alors en préparation du journal inédit de l'helléniste. J'étais accompagné d'un ami athénien qui ignorait tout du tsakonien. Je n'oublierai jamais la stupéfaction et le bonheur des gens, m'entendant balbutier des mots dont j'avais précisé qu'ils avaient été recueillis à la fin du XVIII siècle, des mots qui étaient toujours en usage et que mon ami athénien se faisait aussitôt traduire dans ce qu'il appelait « sa » langue, le grec commun. 


\section{NOTES}

1. Sur l'institution des tsakones, cf. d'une manière générale, S.C. CARATZAS, Les Tzacones, Berlin, 1976.

2. Selon CARATZAS, o.l., p. 166-167, l'institution a dû connaître un développement important sous les règnes de Constantin V (770-775) et de Nicéphore Phocas I (802-811), qui ont beaucoup œuvré au renforcement des fortifications des frontières, à la suite, notamment, d'une vague d'immigration slave en Morée.

3. Le moment essentiel de cette redécouverte est l'ouvrage de M. KRAUS (CRUSIUS), Turco-Graeciae libri VIII, Bâle, 1584. Pour un examen des quelques témoignages antérieurs (Eustathe, témoignages relatifs à la Grèce sous l'empire ottoman), cf. CARATZAS, o.l., p. 232-239.

4. CARATZAS, o.l., p. 5-38, passe en revue toutes les étymologies proposées.

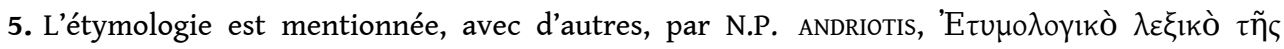

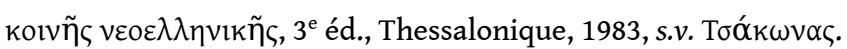

6. Cf. CARATZAS, o.l., p. 281-309.

7. Selon un recensement de 1983, le tsakonien est encore pratiqué par 8500 personnes environ.

8. Sur le personnage et les circonstances de son voyage, cf. É. FAMERIE, Jean-Baptiste-Gaspard d'Ansse de Villoison. De l'Hellade à la Grèce. Voyage en Grèce et au Levant (1784-1786), Hildesheim, 2006. L'ouvrage contient, entre autres, l'édition princeps du journal de voyage.

9. «Aux cuisses découvertes » (épithète appliquée par le poète Ibycos aux jeunes filles de Sparte, selon PLUT., Comp. Lyc. et Numa, 3, 6).

10. Cf. FAMERIE, o.l., p. 52.

11. Les papiers de Villoison sont conservés à la BnF (Suppl. gr. 929-966, 989, soit 39 volumes) : cf. Ch. ASTRUC - M.-L. CONCASTY, Bibliothèque Nationale. Département des manuscrits. Catalogue des manuscrits grecs. Troisième partie. Le Supplément grec, t. III, Paris, 1960.

12. Cf. FAMERIE, o.l., p. 124.

13. Cf. FAMERIE, o.l., p. 229-233.

14. Venise, 1788, p. XLIX-L, n. 1. Pour donner un aperçu de la méthode de travail de Villoison, j'ai conservé, dans des notes de bas de page, les références bibliographiques et les passages parallèles fournis par l'auteur dans son exposé. Sur la préface en général, cf. FAMERIE, o.l., p. 21-22.

15. Sic, teste Salmasio in notis ad Consecrationem templi in agro Herodis, p. 48, ed. Paris. 1619, Aeoles

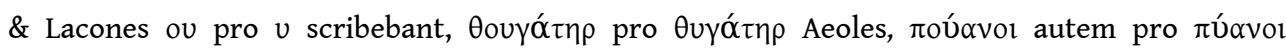

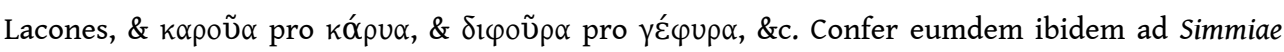
Rhodii ovum, p. 176, ubi Corinnae fragmentum laudat, \& in Commentario de Hellenistica, p. 77 ; Eustathium, p. 23, t. I ; fragmentum Corinnae apud Hephaestion., p. 60, \&c.

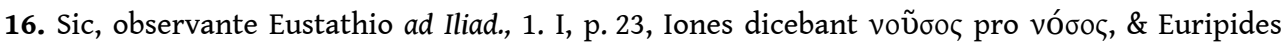

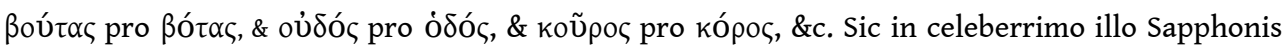

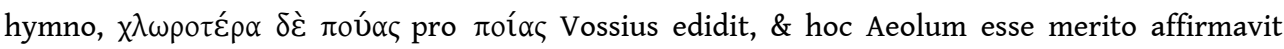
summus ille Brunck ad hunc versum 12, p. 137 ed. Anacreontis, Argentorati, 1786. Vide Salmas., p. 18 \& 36 notar. ad Consecration. templi in agro Herodis.

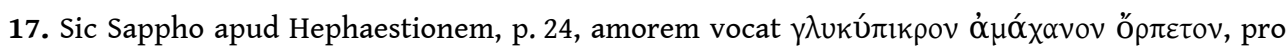

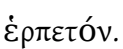

18. Vide Koen ad Gregor., de dialectis, p. 80.

19. Hinc repetenda illa Homerica forma tam frequens, \& quae tamdiu Graecos torsit, i $\pi \pi$ ó $\alpha$

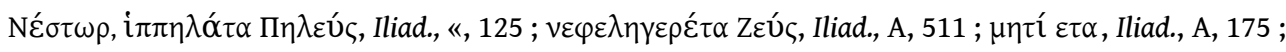

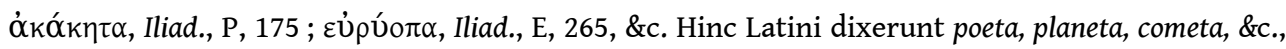
hanc formam sequuti. 
20. Fallebatur igitur Salmasius, cum haec scribebat de Hellenistica, c. 8, p. 447: Eo postremo deventum est temporis progressu, ut tollerentur omnes dialectorum differentiae apud Graecos, et uniformis facies Graeci sermonis per universam Graeciam diffunderetur, eaque corruptissima. Si quis hodie in vulgari Graeca requireret dialectorum illam quadripartitam varietatem quas olim obtinuit, Iadis, Atthidis, Doridis et Aeolidis, frustra esset. A multis retro saeculis ea sublata et abolita linguarum discrimina constat ... Graeci grammatici, cum exempla referenda sunt Ionicae, aut Doricae, vel Aeoliae Atticaeve dialectorum, non dicunt eas dialectos vigere in Attica, Aeolide, Ionia vel Doride, nec sane poterant, \&c., \&c. Iam supra observavimus nostrum interpretem Venetum ad Iliad., O, 536 \& 545, \& P, 117, Aeolicae \& Ionicae dialectorum, tamquam adhuc suo tempore, ع̌ $\tau$ $\check{\varepsilon} \omega \varsigma$ ṽ̃v, vigentium mentionem facere. Illam prope Taurum Montem Heracleam non adivi, prope quam amplum esse pagum a solis habitatum christianis Graecis, quorum lingua vulgaris pura Graeca est, adfirmat Bellonius, c. CXI Observation., p. 391 versionis Latinae editae Antuerpiae, 1589.

21. La bibliographie la plus complète est accessible sur le site http://www.tlg.uci.edu/ opoudjis/ Work/tsakbib.html. Quelques références historiques ou fondamentales: G. DEVILLE, Étude du

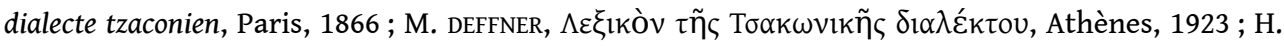

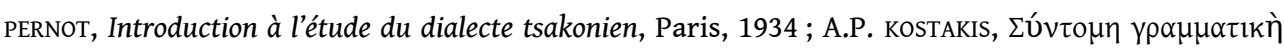

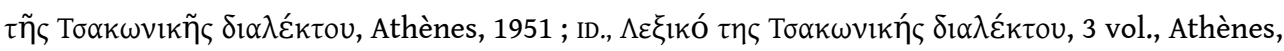
1986-1987.

22. Les projets annoncés par Villoison sont innombrables : cf. FAMERIE, o.l., p. 12-13. Comme tant d'autres, il n'a probablement jamais été mis en chantier. Hormis les quelques pages du journal, rien de tel, en tout cas, ne se trouve dans ses papiers conservés à la BnF.

23. Villoison, comme bien d'autres après lui, a été confronté à l'impossibilité de noter avec précision les sons particuliers en s'en tenant à l'alphabet grec traditionnel (j'adopte le système

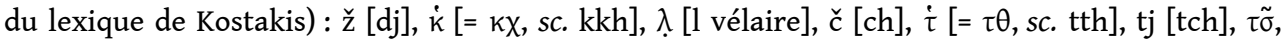
entre [tch] et [ts].

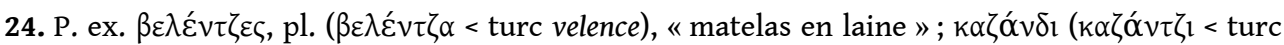

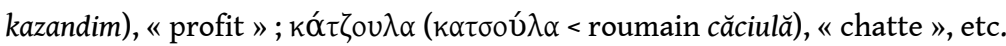

25. Dans le journal manuscrit, la difficulté de la notation est souvent contournée par le recours à

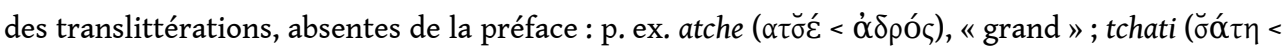

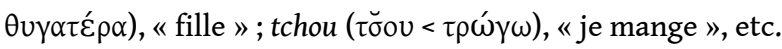

26. DEVILLE, o.l., p. 30 ; C.A. scUTt, The Tsakonian Dialect, dans ABSA, 19 (1912-1913), p. 140, n. 40 ;

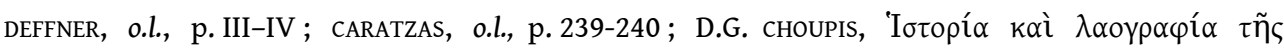

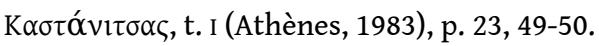

27. Cf. FAMERIE, o.l., p. 10-11.

28. A History of Classical Scholarship, t. II ${ }^{3}$ (Cambridge, 1921), p. 398 («the last scholar of the old school »).

\section{AUTEUR}

\section{ÉTIENNE FAMERIE}

Université de Liège

efamerie@ulg.ac.be 